\title{
Deep Learning-Based Self-Driving Car: JetBot with NVIDIA AI Board to Deliver Items at Agricultural Workplace with Object-Finding and Avoidance Functions
}

\author{
Shinji Kawakura and Ryosuke Shibasaki
}

\begin{abstract}
In this study, we attempt to develop a deep learning-based self-driving car system to deliver items (e.g., harvested onions, agri-tools, PET bottles) to agricultural (agri-) workers at an agri-workplace. The system is based around a carshaped robot, JetBot, with an NVIDIA artificial intelligence (AI) oriented board. JetBot can find diverse objects and avoid them. We implemented experimental trials at a real warehouse where various items (glove, boot, sickle (falx), scissors, and hoe), called obstacles, were scattered. The assumed agri-worker was a man suspending dried onions on a beam. Specifically, we developed a system focusing on the function of precisely detecting obstacles with deep learning-based techniques (techs), self-avoidance, and automatic delivery of small items for manual agri-workers and managers. Both the car-shaped figure and the deep learningbased obstacles-avoidance function differ from existing mobile agri-machine techs and products with respect to their main aims and structural features. Their advantages are their low costs in comparison with past similar mechanical systems found in the literature and similar commercial goods. The robot is extremely agile and easily identifies and learns obstacles. Additionally, the JetBot kit is a minimal product and includes a feature allowing users to arbitrarily expand and change functions and mechanical settings.
\end{abstract}

This study consists of six phases: (1) designing and confirming the validity of the entire system, (2) constructing and tuning various minor system settings (e.g., programs and JetBot specifications), (3) accumulating obstacle picture data, (4) executing deep learning, (5) conducting experiments in an indoor warehouse to simulate a real agri-working situation, and (6) assessing and discussing the trial data quantitatively (presenting the success and error rates of the trials) and qualitatively. We consider that from the limited trials, the system can be judged as valid to some extent in certain situations. However, we were unable to perform more broad or generalizable experiments (e.g., execution at mud farmlands and running JetBot on non-flat floor). We present experimental ranges for the success ratio of these trials, particularly noting crashed obstacle types and other error types. We were also able to observe features of the system's practical operations. The novel achievements of this study lie in the fusion of recent deep learning-based agricultural informatics. In the future, agriworkers and their managers could use the proposed system in real agri-places as a common automatic delivering system. Furthermore, we believe, by combining this application with other existing systems, future agri-fields and other workplaces could become more comfortable and secure (e.g., delivering water bottles could avoid heat (stress) disorders).

Index Terms - Deep learning, identifying objects, avoiding obstacles, delivering items, NVIDIA Jetson Nano, self-driving.

\section{INTRODUCTION}

In recent years, agricultural (agri-) researchers, agrimanagers, and agri-workers have developed a wide range of automatic and mechanical techniques (techs). As a result, productivity, agri-cost, and other factors have improved. In particular, automations have continued to evolve with the combination of informatics, mechatronics, and electric engineering.

Practically, for actual agri-management sites, obtaining and sharing diverse real-time information is a crucial factor directly related to the success or failure of modern agrimanagement. Recent studies in scientific research and business have developed and verified deep learning systems, Internet of Things (IoT) systems, and smart contract-based systems (e.g., blockchain) [1-8].

Specifically, Kamilaris et al. [1] presented the latest techsbased trends in agriculture and food supply fields, focusing on food science and technology. Omar et al. [2] and Cichosz et al. [3] constructed and presented concrete examples of IoT and smart contract techs-based platform systems for health care [2], including practical treatments for diabetic patients [3].

Regarding IoT and smart contracts for diverse vehicles, Liu et al. constructed and presented IoT and smart contractenabled systems aimed at improving security using electric vehicle clouds and edge computing [4], and Cebe et al. looked at a lightweight IoT and smart contract framework for forensic applications of network-connected vehicles [5]. From the perspective of IoT-based security and integrity, Yu et al. presented IoT and smart contract-based solutions to enhance the security and privacy levels of IoT [6]. Machado and Fröhlich verified IoT data integrity concerning cyberphysical systems using blockchain-based systems [7]. With respect to robotics, Strobel et al. managed byzantine robots with IoT and smart contract techs for a swarm robotics decision-making scenario [8].

There are several more closely related studies looking at high-tech systems for agri-research and business-based management [9-13]. Fukatsu et al. [9-10] designed, developed, and handled web-based sensor systems. They used systems with Field Servers for practical agricultural 
applications to obtain diverse data from real outdoor agrifields [10]. Additionally, Brun-Laguna et al. demonstrated the PEACH IoT-based frost event prediction system with consideration of recent precision agri-techs [12]. Karim and Karim [13] constructed and managed a monitoring system using Web of Things (WoT). Several studies have collected and analyzed diverse human or robot physical data [14-18]. Zhao et al. executed and presented a model targeting agrirobotics based on an acceleration sensor [14]. Some research has employed deep learning [15-18]. For example, Orii et al. recognized tactile textures using recent deep learning-based methodologies (convolutional neural networks (CNNs)) for time-series data of pressure and six-axis acceleration sensors [15]. Dawar and Kehtarnavaz detected and recognized human continuous streaming actions using deep learning-based sensing fusions [16]. Jiang and Yin recognized human activities from wearable sensors using deep CNNs [17], similar to Hassan et al. [18].

Jetson Nano and JetBot are two recent, strong and valid infrastructures that allow the execution of diverse deep learning based trials and the collection of useful, meaningful data concerning real-world objects and fields [19-23].

In this study, considering the aforementioned literature, technical backgrounds and trends, we selected Jetson Nano and JetBot (JetBot AI kit with NVIDIA Jetson Nano: Selfdriving learning set). Jetson Nano was designed, constructed and distributed by NVIDIA Inc., California, USA. JetBot was designed, constructed and distributed by XiaoR Geek Inc., USA. JetBot is a two-wheeled car-shaped system that mounts the Jetson Nano board and other peripheral parts like sensors and a camera. We describe the specifications in later chapters.

We developed and attempted to implement a deep learning Jetson Nano and JetBot-based system utilizing agri-visual records for traditional, physical agri-workers, and managers. We suggest novel deep learning-based obstacle avoidance approaches for practical agri-managers and workers, as presented in Fig. 1. For these cohorts, special information utilizing technologies and multiple sensors could change skill requirements and enhance security levels.

We present the following advantages of the proposed approach:

1) The system is low cost compared with past similar mechanical systems developed by academic researchers and other similar commercial goods.

2) The system is extremely agile and easily identifies and learns obstacles.

3) The JetBot kit is a minimal product, so it includes a feature that allows users to arbitrarily expand and change functions and mechanical settings.

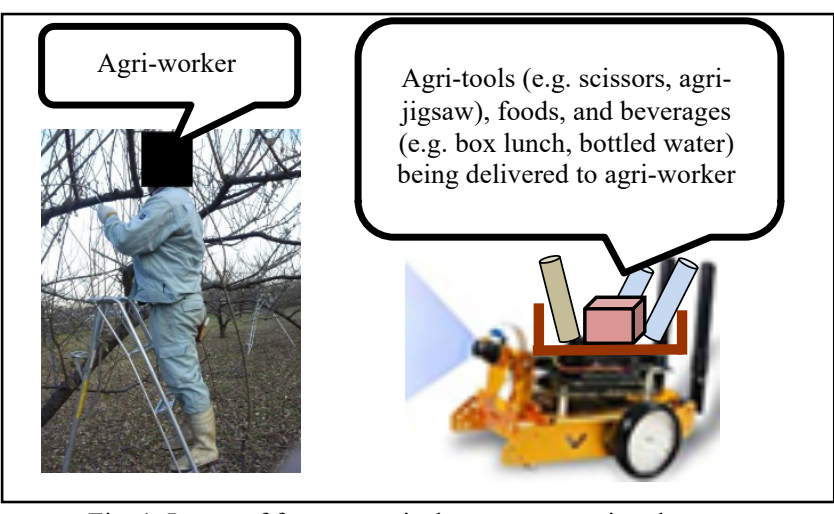

Fig. 1. Image of future practical usage concerning the system.

\section{METHOD}

\section{A. Overview}

This study consists of five phases: (1) designing and confirming the Jetson Nano and JetBot based system, (2) constructing and tuning the system settings (e.g., picture data accumulation and deep learning programs), (3) conducting experiments at a real agri-field; in this study, we select and use a non-specific, small to middle sized warehouse, (4) observing and estimating the system's performance, and (5) discussing the future.

As presented in Fig. 1, this study has two fundamental functions to accomplish our aims: (A) automatically going to a location near a working agri-worker's foot from a sign (marker) on a neutral position in a flat, asphalt-paved (bituminous pavement) warehouse, and (B) avoiding objects after deep learning (in short, a general collision avoidance function)

For these functions, we present the following simplified flow:

(1) Construct and set up the tools and fields before the main tasks. We performed a literature review to identify research and commercial achievements. Then, we designed systems for practical execution in indoor warehouse settings and farmlands. We built the system and verified which components are essential for operation.

(2) Gather and tag visual data as (A) "no problem data: no need to avoid" in the "free folder" or (B) "obstacle data: need to avoid" in the "blocked folder" (collection datasets). These datasets are then split into training and test datasets.

(3) Train the neural net for deep learning and create a pretrained "alexnet model (AlexNet)" file (training model) with batch size $=16$, shuffle $=$ True.

(4) Upload the trained model to the Jetson Nano board mounted on the JetBot.

(5) Execute the practical experiment.

\section{B. JetBot-based System}

In this trial, we used a JetBot artificial intelligence (AI) kit with a NVIDIA Jetson Nano-based rearranged system. No agri-research has previously utilized the system, but some research has been undertaken in educational methodologybased research fields.

The fundamental components for these specifications are the Jetson Nano microcomputer board and the NVIDIA Jetson Nano Developer Kit, which is the packaged, twowheeled car-shaped experimental system kit set developed and distributed by NVIDIA Inc. 
The aforementioned board is used in the areas of executing diverse deep learnings, such as image data classifications, detecting indicated objects, automatic segmentation computing, recognizing diverse sound data including real human voice data, and parallel processing for some of the above.

Jetson Nano runs on a specific operating system (OS) called Linux4Tegra OS, and the program development environment is a Jetpack software development kit (SDK). The SDK contains the aforementioned OS, general library, application programming interface (API) files, developer tools, documents, demo-samples, Compute Unified Device Architecture (CUDA), and Robot Operating System (ROS).

The CUDA Toolkit is a development environment to develop high-performance graphical processing unit (GPU)accelerated applications. Developers can create and optimize diverse applications on GPU-accelerated systems. It can be used flexibly for desktop workstations, enterprise data centers, cloud-based platforms, and high-performance computing (HPC) supercomputers. CUDA sets include libraries, debugging and optimization tools, a compiler, and a runtime library to deploy applications. ROS is a set of software libraries to help developers build robot-based applications. It includes drivers, state-of-the-art algorithms, and powerful developer tools, and is open source.

We describe other specifications of the components of the JetBot-based system below. A small-sized, 8 MP 160 FOV, camera was installed with a resolution of $3280 \times 2464$ pixels.

For the object-detecting sensor, we used a Sony IMX 219 sensor. For the microcomputer board, we used the widely available Jetson Nano board, an alternating current (AC) electrical resource set, an HDMI cable, and jumper pins, all distributed by NVIDIA Inc.

For data storage, we must use a common (non-specific) Micro SD card; thus, we used a 64 Gigabyte card. An organic light-emitting diode (OLED: small-sized monitor board) was mounted with a monitor size of 0.91 inch, $128 \times 32$ pixels, so the IP address, RAM, and battery life could be displayed. Two dual-mode wireless NIC AC8265 antennas, which are relatively high speed, were installed to connect the kit with Wi-Fi.

This relatively compact and simple composition allows flexible redesign (e.g., attaching other sensors or IoT modules).

For the deep learning process, we must change the electricity consumption settings because the process has high consumption. Thus, we apply the conditions of " $20 \mathrm{~W}(5.0 \mathrm{~V}$, 4.0 A) mode" instead of " $5 \mathrm{~W}$ mode" and change the Power Mode from the default setting to "MAXN mode" in advance.

\section{Deep learning-based Process}

We programmed the system codes on the online platform Google Collaboratory, a domain online platform. For some parts, we used a Jupiter and Anaconda based platform (Fig. 2 ). Several studies have used open source codes for various tasks (e.g., arbitrary objective tracing and running avoiding obstacles indicated in advance, simple object-identifications).

For the neural network for deep learning, we select and use an "alexnet model (AlexNet)" file format. AlexNet is a CNN) with several layers; the first five are convolutional layers, followed by max-pooling layers, and the final three are connected layers.
We used the non-saturating rectified linear unit (ReLU) activation function that presents improved training performance over tanh and sigmoid. AlexNet is considered one of the most dominant neural networks in vision computing fields and has been used for splendid studies employing CNN and GPU.

For the definition of the neural network, since the AlexNet model exists in the Torchvision Package, we changed the last layer that has only two data-outputs into a new layer that is non-trained.

According to trends in related studies, we executed the training through 30 epochs; set batch size $=16$, shuffle $=$ True, Optimizer $=$ SGD (Stochastic Gradient Descent), Ir $=0.001$, and momentum $=0.9$. SGD is an iterative method for optimizing an objective function with smoothness properties. It can be regarded as a stochastic approximation of gradient descent optimization. It replaces the actual gradient by an estimated gradient calculated from a randomly selected subset of the data. Especially in large-sized data applications, it can reduce computational burdens, achieving faster iterations in exchange for a slightly lower convergence rate.

We selected and utilized the sigmoid function, which is typically used as an activation function for neural networks in this academic field.

Next, we loaded the trained model on the system. For the first phase, we imported PyTorch, torchvision, OpenCV2, and numpy. However, the trained model's format does not exactly fit the format of the camera installed on the JetBot. Thus, we executed the following preprocessing steps:

(1) Convert color codes from blue-green-red (BGR) into redgreen-blue (RGB),

(2) convert from height-width-channel (HWC) layout into channel-height-width (CHW) layout,

(3) normalize the parameters to those used in the training phase,

(4) transfer the data from the central processing unit (CPU) memory into GPU memory, and

(5) add a batch dimension.

For the object detection phase, we utilized the You Only Look Once (YOLO) algorithm for object detection [22][24][25] (Figs. 3 and 4). In particular, we applied Tiny YOLO version 2 , which consists of nine convolutional (conv) layers.

To utilize a Five Anchor Box-based method, we input pictures of size $416 \times 416$ pixels. We used a general methodology to calculate the bounding boxes. With an assumed five anchor boxes, predicted values are output related to each one square of the grids. To identify bounding box candidates (proposing bounding boxes), there are five predictive values as well as 20 classes of existence probabilities to be directed in this case.

The grid-size of input data related to Tiny YOLO version 2 is basically $13 \times 13$. The number of anchor box (previously formed standard bounding box) is five, and for the use of Tiny YOLO version 2, specific predicted values are output assuming five anchor box per aforementioned each grid respectively; These predicted values consist of $i$ ) five numbers to present the candidates of bounding boxes, and ii) the data of existence probabilities concerning each twenty classes. Therefore, the number of all factors concerning network outputs is calculated as $13 \times 13 \times 5 \times(5+20)=$ 21,125 .

In line with bounding box-based theory, two or more bounding boxes may be detected concerning a single 
objective (Fig. 3). In such a case, we apply Non-Maximum Suppression (NMS). That is, we select and use only the bounding box with the maximum size, and we eliminate the other bounding boxes. Further, we use the Intersection over Union ( $\mathrm{IoU}$ ) index to estimate the range of the bounding boxes' overlapping. Specifically, we select and use one rectangle that is related to the maximum IoU. Regarding these techniques, Girshick et al. noted, "Given all scored regions in an image, we apply a greedy non-maximum suppression (for each class independently) that rejects a region if it has an intersection-over-union (IoU) overlap with a higher scoring selected region larger than a learned threshold" [26-29].

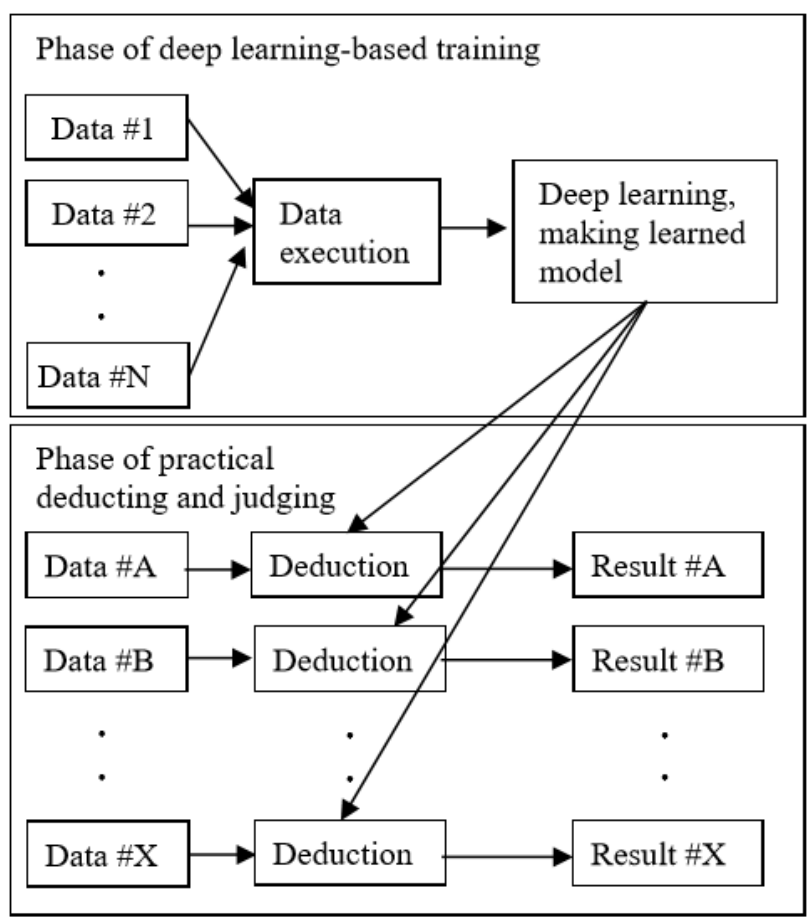

Fig. 2. Overview of the deep learning-based process.

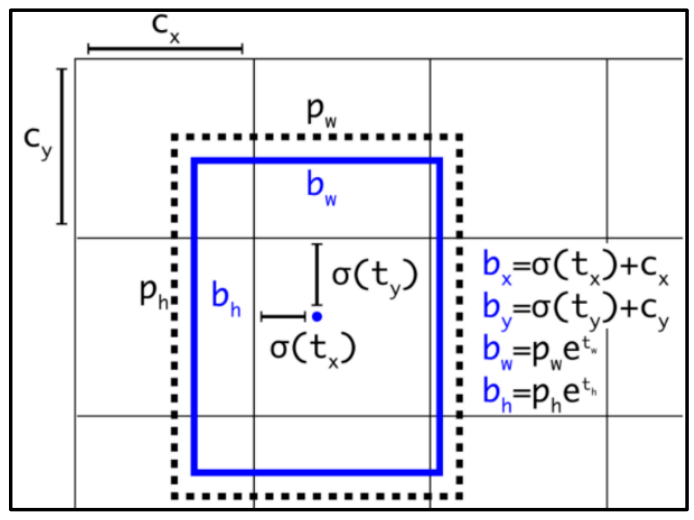

Fig. 3. Bounding boxes with dimension priors and location prediction [24]: We predict the width and height of the box as offsets from the cluster centroids. We predict the center coordinates of the box.

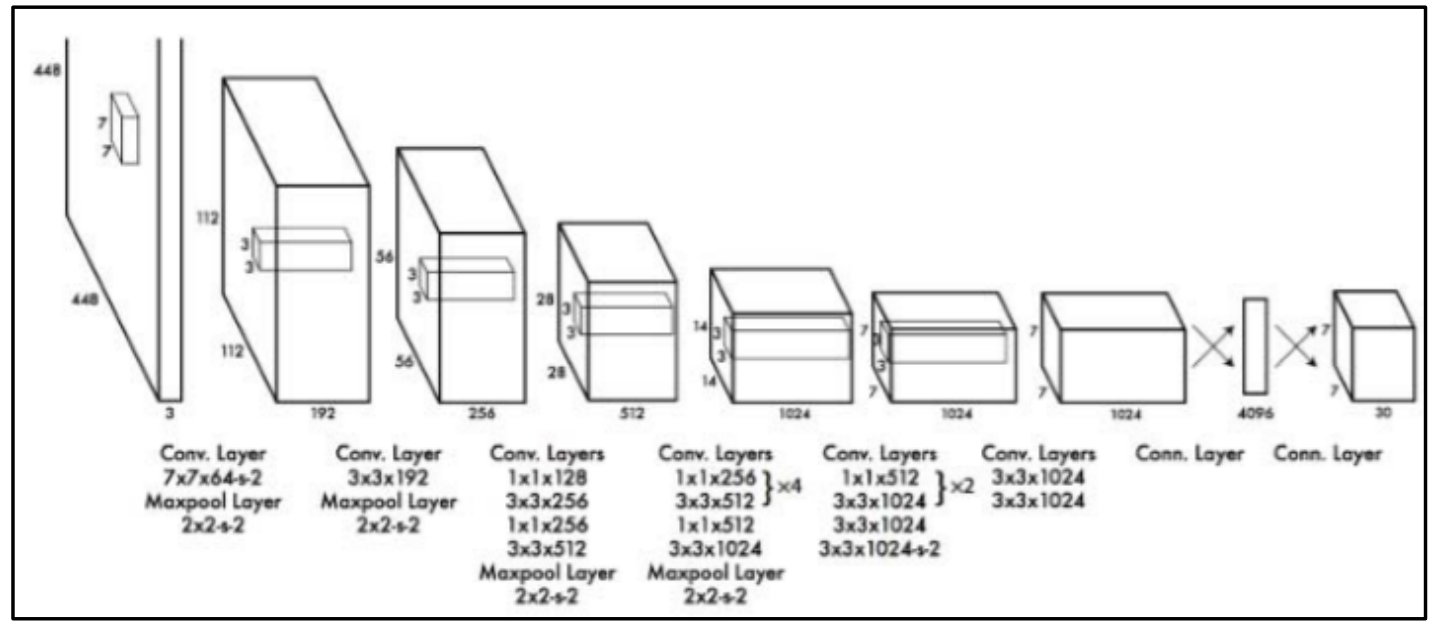

Fig. 4. You Only Look Once architechture: Unified, Real-time Object Detection [25].

For the practical deduction and judging component, we used the Open Neural Network Exchange (ONNX) format, which was researched and developed mainly by Microsoft Inc. and Facebook Inc. (California, U.S.A.) for a special community project. These companies have been developing and providing pre-learned models.
After the model file is converted into the ONNX format, it can be reused between diverse deep learning frameworks. We use the neural network model at it is easier to adapt than other methodologies. 
Users can utilize many kinds of openly available prelearned deep learning model data created in the ONNX format, provided on the specific website for developers.

In this study, we do not use an existing, pre-learned .pth format file (pth-file), but create an original file. For deep learning, we apply the following two picture datasets: (1) a dataset concerning the situation in which JetBot can go forward, and (2) a dataset concerning the situation in which JetBot should change the direction of movement. We utilize them in successive phases and make a network .pth-file for pre-learned file-based deep learning.

Two factors are crucial:

(1) Executing deep learning processes with a picture dataset obtained by the installed camera on JetBot, and

(2) creating the .pth file.

After the pre-training, we execute outdoor experiments

\section{Experimental Method}

We developed the JetBot-based system to move and avoid various obstacles at a non-specific warehouse (Figs. 5-8). Then, we executed practical experiments to test the system's performance, especially in relation to obstacle avoidance.

Here, the system automatically moves through the nondirected path between positions $B$ and $G$ (via a path from $B$ to $G$, or from $G$ to $B$ ) shown in Figs. 6 and 7, assuming (simulating) the situation where the system is sending diverse items (harvested vegetables, agri-tools, PET bottles, etc.) placed in the box mounted on the JetBot's body. JetBot has previously input a command to go to the right automatically if it is about to crash into walls or other non-directed items (i.e., one of the \#1-5 obstacles).

The obstacles (target objects to avoid collisions with) are shown, including descriptions, in Fig. 5. The obstacles are \#1 Glove, \#2 Boot", \#3 Sickle, \#4 Scissors, and \#5 Hoe. These obstacles were selected based on the opinions of real agrifarmers and other social information (e.g., academic papers concerning agriculture, guidance books for agri-newcomers), as they are rather common agri-tools. Thus, we make much of the generality and real opinions from experienced agrifarmers and managers of common farms with 5-20 years of experience. That is, our results are not limited to the experimental conditions but could be applied in various specific agri-situations.

JetBot is $190 \mathrm{~mm}$ long, $125 \mathrm{~mm}$ wide, and $105 \mathrm{~mm}$ high, excluding the two antennas to connect to Wi-Fi networks. It also has a rubber wheel that is $60 \mathrm{~mm}$ in diameter.

The warehouse is $5400 \mathrm{~mm} \times 5200 \mathrm{~mm}$ (Fig. 6). Position $A$ is the area of accumulated harvests, Position $B$ is the neutral position for Jetson, Positions $C-E$ have agri-tools (e.g., scissors and ladders), Positions F1-5 contain obstacles, and Position $G$ is near the agri-worker's foot. The yellow circle indicates the starting position's marker and the red circle indicates the goal position's marker (Figs. 6, 7, and 8); this JetBot will run between these markers. We intentionally selected and used vivid and distinct balls as markers, with consideration for characteristics in related studies.

We set the obstacles with the same margins as much as possible, since the experimental results would not be universal if these obstacles were grouped together instead of scattered with an equal distance. Note that in the deep learning phase, we used five patterns to scatter the five types of obstacles. utilizing the pth-file.

We must uniform the mechanisms and their settings between (1) the process of gathering picture data for the deep learning and (2) the process of outdoor experimental trials. We also must align the conditions of the experimental fields where the JetBot runs to ensure accuracy and consistency for the trials.

In the JetBot kit's SD card, Python, PyTorch, and CUDAbased notebooks for obtaining picture data are pre-loaded for general users. We decide to utilize these with consideration for the current technical situation and trends. For the graphic computing, we used a GPU instead of a CPU.

Before the learning, we split the picture dataset into training and test sets. The test dataset is used to asset the performance of the trained model.

We describe the detailed methodology of image data gathering below. We consider the images as pre-trained model file data. We captured 100 picture data total by photographing each of the five obstacles 20 times (10 times each for "no problem data" and "obstacle data"). We rotated the camera angle at intervals of 18 degrees $(360 \div 20=18)$, beside the two markers on the starting position and god position (yellow and red balls, presented in Figs. 6 and 8).

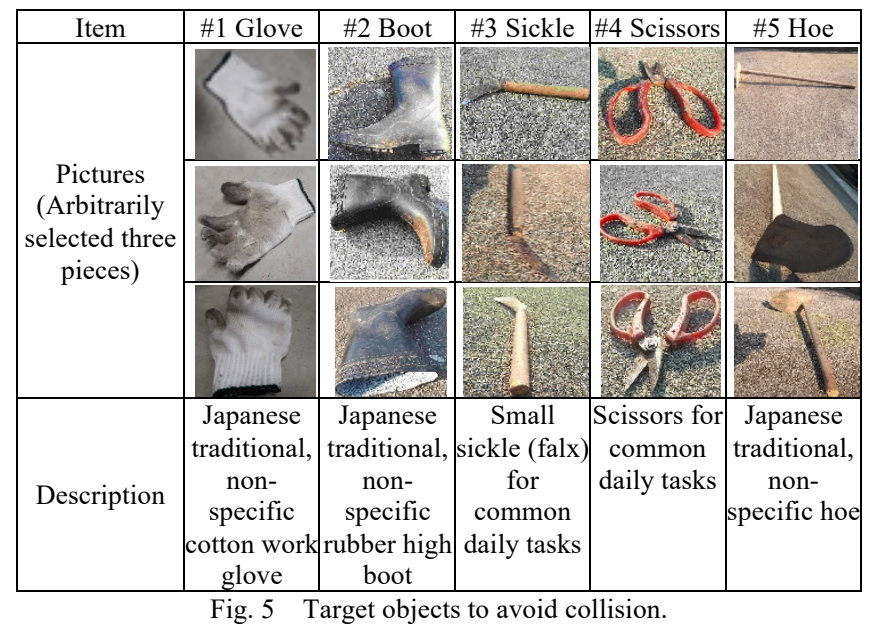

The actual experiment phase is conducted by (1) using the aforementioned previous settings (indoor fundamental functions' checking of JetBot), (2) setting practical probabilities that the JetBot will avoid obstacles according to the amount and angle of light (light intensity) at the warehouse, and (3) running test trials, with consideration for future practical studies at the same warehouse, such that this model could become a promising, practical system in polymorphic, real agri-fields all over the world (Fig. 9).

Specifically, for (1), we set the JetBot-based system on position $B$ near the yellow marker (and place accumulated dried onions accumulated on the position $A$ ), and push the start-button on the system.

In the event of unexpected problems during the trials, the experimenter stops the movement of the system by hand and turns off the main button of the system. Otherwise, the trial ends when the JetBot reaches the red marker. 


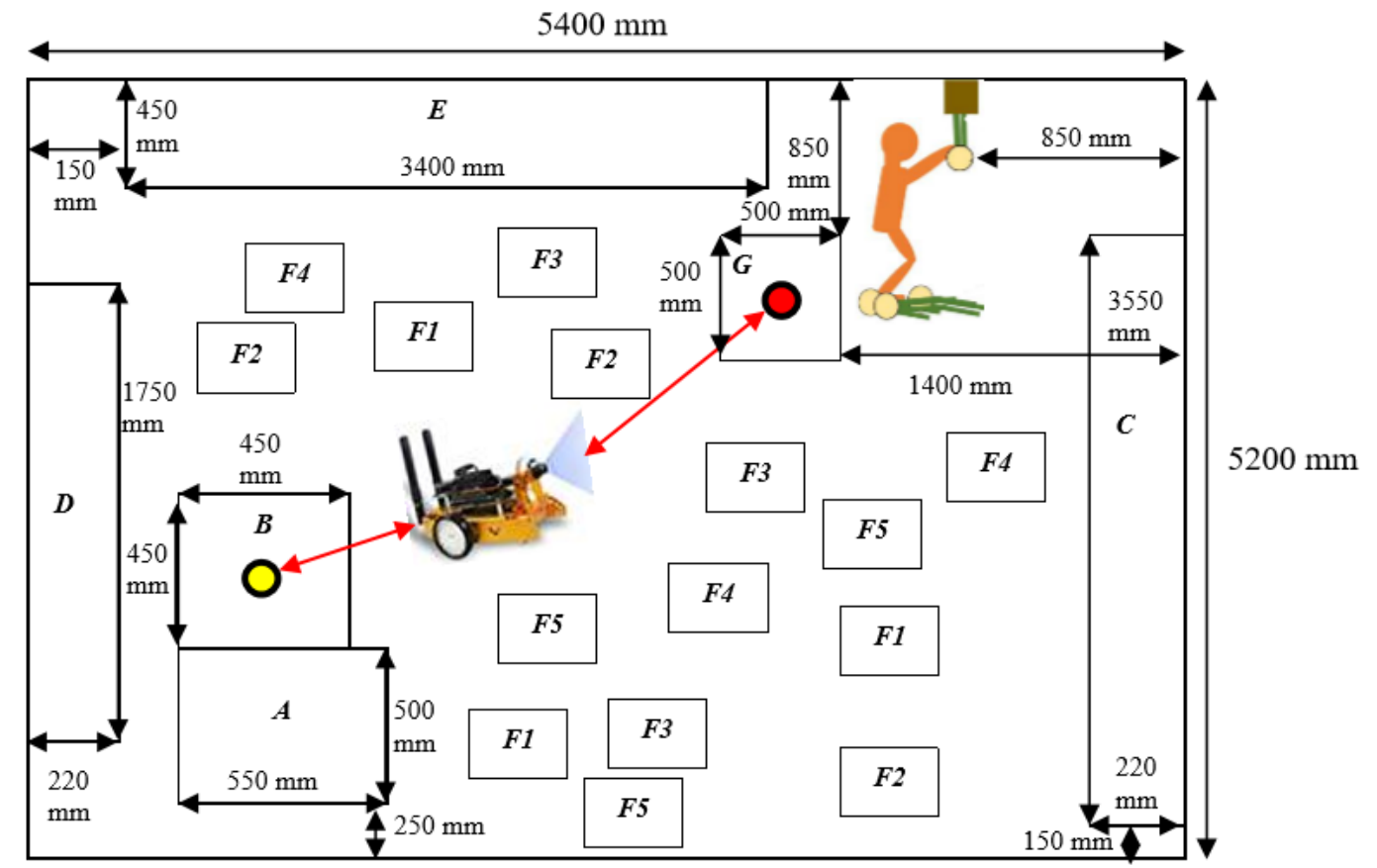

Fig. 6. Image of experimental field: non-specific, Japanese traditional warehouse to store agri-tools, harvests, and other items.

(Position $A$ : accumulated harvests, $B$ : neutral position for Jetson (the yellow circle is the starting position's marker),

$C$ - $E$ : agri-tools (e.g., scissors, ladders), $F 1-5$ : scattered obstacles (\#1-5, respectively),

and $G$ : near the agri-worker's foot (the red circle is the goal position's marker).)

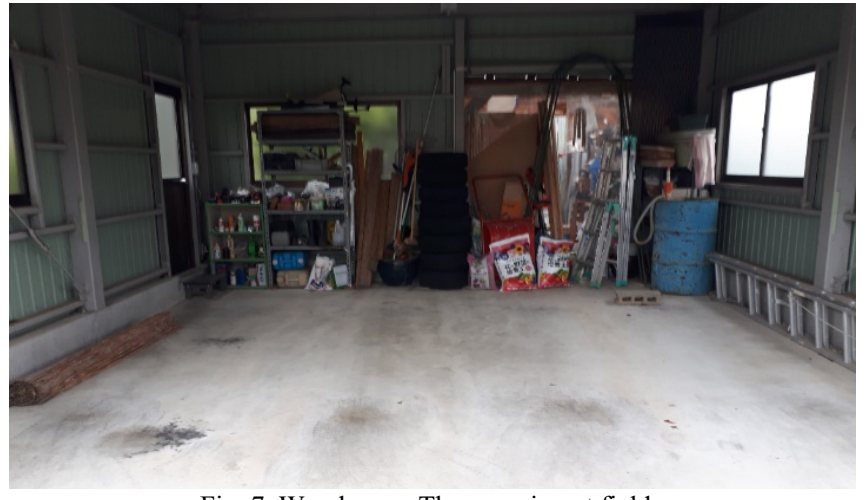

Fig. 7. Warehouse: The experiment field.

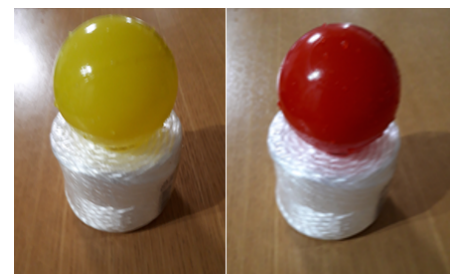

Fig. 8. Markers on the starting potiton and goal position, as shown in Fig. 6.

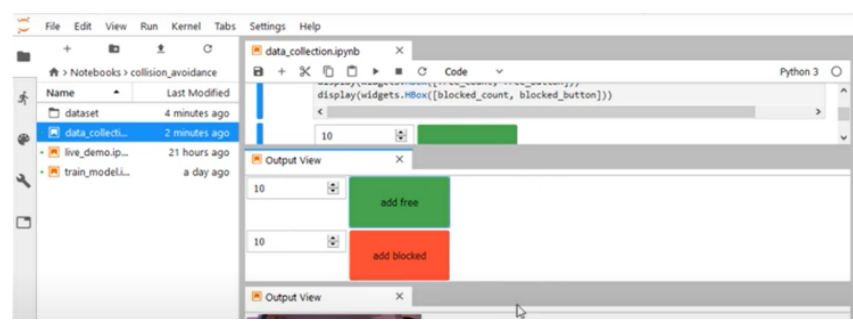

Fig. 9. A window concerning picture-data collection and the deep learning phase.

\section{RESULTS}

First, we define three cases: (1) complete success (completely appropriate movement) of the trial is the case where JetBot runs from position $B$ to $H$, or vice versa, avoiding all obstacles appropriately, (2) success (appropriate movement) of the trial is the case where JetBot could run from position $B$ to $H$, or vice versa, generally avoiding obstacles appropriately but crashing into some obstacles, (3) failure (invalid movement) of the case where JetBot touches or crashes into obstacles $A, C, D, E, F 1-5$, or walls of the warehouse and cannot reach the aimed position.

Second, we present experimental trial data (average value and standard deviation (S.D.)) in Table I for Successful case ratio (\%), Trial time (number), Collision Count (number), Collided Objects (Object \#, number of times), and Execution Time (s).

The successful case ratio (the cases of Complete Success or Success divided by the total cases) was (a) $56.2 \%$ from position $B$ to $H$, and (b) $69.0 \%$ from position $H$ to $B$.

As is usual in such studies, in some cases, the system could not run mainly due to detection errors. For instance, this could include the following three cases: (1) JetBot cannot detect obstacles quickly enough so it cannot avoid them, (2) JetBot detects obstacles too early, and (3) JetBot cannot detect obstacles for mechanical or other reasons.

Further, there may occasionally be unexpected situations and accidents, such as in cases where, after avoiding obstacles, Jetbot's wheels get stuck on or spool up obstacles. 
TABLE I: EXPERIMENTAL DATA FOR JETBOT BASED TRIALS (AVERAGE, (S.D))

\begin{tabular}{|c|c|c|c|c|}
\hline $\begin{array}{l}\text { Success ratio } \\
\text { (complete } \\
\text { success or } \\
\text { success) }(\%)\end{array}$ & $\begin{array}{l}\text { Trial } \\
\text { Time } \\
\text { (numbe } \\
\text { r) }\end{array}$ & $\begin{array}{l}\text { Collision } \\
\text { Count } \\
\text { (number) }\end{array}$ & $\begin{array}{l}\text { Collided Objects } \\
\text { (Object\#, number } \\
\text { of times) }\end{array}$ & $\begin{array}{l}\text { Executio } \\
\text { n Time } \\
\text { (s) }\end{array}$ \\
\hline (a) from & & & $\# 10.987$ (0.123) & \\
\hline $\begin{array}{l}\text { position B to } \\
\mathrm{H}\end{array}$ & 5 & $\begin{array}{c}8.80 \\
(4.23)\end{array}$ & $\begin{array}{ll}\# 2 & 0.355(0.168) \\
\# 3 & 2.82(2.64) \\
\# 4 & 1.07(0.101) \\
\# 5 & 3.04(0.961)\end{array}$ & $\begin{array}{c}81.8 \\
(33.1)\end{array}$ \\
\hline $\begin{array}{l}\text { (b) from } \\
\text { position } \mathrm{H} \text { to } \\
\text { B }\end{array}$ & 5 & $\begin{array}{c}7.29 \\
(6.79)\end{array}$ & $\begin{array}{ll}\# 1 & 0.852(0.556) \\
\# 2 & 0.948(0.388) \\
\# 3 & 4.96(2.21) \\
\# 4 & 0.412(0.280) \\
\# 5 & 3.95(1.36)\end{array}$ & $\begin{array}{l}123.2 \\
(44.9)\end{array}$ \\
\hline
\end{tabular}

\section{DISCUSSION}

In this study, we applied an integrated system with a certain level of quality for real agri-workers at a warehouse. As presented in Section III, we obtained the ratios of successful to total cases and described the characteristics of the system's operation.

We could not find past precedents for this approach in the literature or commercially. Thus, quantifying the data accuracy is difficult, and we experimented only in limited conditions.

As is typical in related studies, the system was not always successful. For example, detection errors could prevent a successful case if (1) JetBot cannot detect obstacles fast enough and so cannot avoid them, (2) JetBot detects obstacles too early, or (3) JetBot cannot detect obstacles for mechanical or other reasons. These situations are serious problems to consider because JetBot cannot recognize or avoid prelearned obstacles for diverse reasons.

For chromatic aberration (chromatism) problems in the captured picture dataset, we leave room for later improvement and could not numerically determine the impact of chromatism. Ideally, in subsequent studies, we should select other high-spec cameras with a wider-angled lens and lower chromatism.

\section{CONCLUSION AND Future TASKS}

We developed and verified fundamental phases of the JetBot and deep learning-based, car-shaped system and methodologies by experimenting in an actual agricultural workplace with Japanese traditional, physical agri-workers. We presented significant and practical quantitative and qualitative data, including output-based suggestions for agricultural leaders, managers, and newcomers.

We described collision-avoidance functions and characteristics concerning movement. However, for methodological reasons, we executed the experimental trials under limited conditions and methodology.

In the future, we plan to run a wholly Internet-connected, scientifically integrated car-shaped system in real agri-fields (e.g., muddy farmland, meadows, and sandy areas) and add more practical functions (e.g., automatic item-dumping system at directed places, automatic siren, and lightning functions).

In this study, we used one system; however, we should try two or more systems in future experiments. Additionally, we should determine the accuracies of such systems qualitatively and quantitatively.

Further, we should re-consider, re-arrange, and change various factors (e.g., the quality of parts, the construction of deep learning program codes). We could also attach various covers to avoid mud, small stones, dusts, etc.; change the tire into off-road oriented tires; and try these kinds of trials at outdoor, muddy agri-fields, or on asphalt pavements (bituminous paved) areas.

We believe that our results have largely achieved a fusion of agricultural informatics, particularly machine learningbased techs, and human dynamics. Furthermore, combined with other agricultural robots, agri-workers and managers could use this model to enhance security.

\section{ACKNOWLEDGMENT}

Our heartfelt appreciation goes to the members of The University of Tokyo who provided considered support, feedback, and comments for our successive researches and creative activity, and the members of Osaka City University.

\section{REFERENCES}

[1] A. Kamilaris, A. Fonts, and F. X. Prenafeta-Boldú, "The rise of blockchain technology in agriculture and food supply chains," Trends in Food Science \& Technology, vol. 91, pp. 640-652, January 2019.

[2] A. Al Omar, M. S. Rahman, A. Basu, and S. Kiyomoto, "Medibchain: A blockchain based privacy preserving platform for healthcare data," Proceedings of International conference on security, privacy and anonymity in computation, communication and storage, pp. 534-543, December 2017.

[3] S. L. Cichosz, M. N. Stausholm, T. Kronborg, P. Vestergaard, and O. Hejlesen, "How to use blockchain for diabetes health care data and access management: an operational concept," Journal of diabetes science and technology, vol. 13, no. 2, pp. 248-253, March 2019.

[4] H. Liu, Y. Zhang, and T. Yang, "Blockchain-enabled security in electric vehicles cloud and edge computing," IEEE Network, vol.32, no. 3, pp. 78-83, January 2018.

[5] M. Cebe, E. Erdin, K. Akkaya, H. Aksu, and S. Uluagac, "Block4forensic: An integrated lightweight blockchain framework for forensics applications of connected vehicles," IEEE Communications Magazine, vol. 56, no. 10, pp. 50-57, October 2018

[6] Y. Yu, Y. Li, J. Tian, and J. Liu, "Blockchain-based solutions to security and privacy issues in the internet of things," IEEE Wireless Communications, vol. 25, no.6, pp. 12-18, December 2018.

[7] C. Machado, and A. A. M. Fröhlich, "IoT data integrity verification for cyber-physical systems using blockchain," Proceedings of 2018 IEEE 21st International Symposium on Real-Time Distributed Computing (ISORC), pp. 83-90, May 2018.

[8] V. Strobel, E. Castelló Ferrer, and M. Dorigo, "Managing byzantine robots via blockchain technology in a swarm robotics collective decision-making scenario," Proceedings of the 17th International Conference on Autonomous Agents and Multi Agent Systems, pp. 541549, July 2018.

[9] T. Fukatsu, T. Kiura, and M. Hirafuji, “A web-based sensor network system with distributed data processing approach via web application," Computer Standards and Interfaces, vol. 33, no. 6, pp. 565-573, November 2011

[10] T. Fukatsu, and M. Hirafuji, "Web-based sensor network system Field Servers for practical agricultural applications," Proceedings of the 2014 International Workshop on Web Intelligence and Smart Sensing, pp. 1-8, September 2014.

[11] T. Fukatsu, and T. Nanseki, "Monitoring system for farming operations with wearable devices utilized sensor networks," Sensors, vol. 9, no. 8, pp. 6171-6184, July 2009.

[12] K. Brun-Laguna, A. L. Diedrichs, J. E. Chaar, D. Dujovne, J. C. Taffernaberry, G. Mercado, and T. Watteyne, "A demo of the PEACH IoT-based frost event prediction system for precision agriculture," Proceedings of 2016 13th Annual IEEE International Conference on Sensing, Communication, and Networking (SECON), pp. 1-3, June 2016.

[13] F. Karim, and F. Karim, "Monitoring system using web of things in precision agriculture," Procedia Computer Science, vol. 110, pp. 402409, January 2017. 
[14] S. Zhao, Z. Zhang, D. Xiao, and K. Xiao, "A Turning Model of Agricultural Robot Based on Acceleration Sensor," IFAC-Papers On Line, vol. 49, no. 16, pp. 445-450, January 2016.

[15] H. Orii, S. Tsuji, T. Kouda, and T. Kohama, "Tactile texture recognition using convolutional neural networks for time-series data of pressure and 6-axis acceleration sensor," Proceedings of 2017 IEEE International Conference on Industrial Technology (ICIT), pp. 10761080, March 2017.

[16] N. Dawar, and N. Kehtarnavaz, "Action detection and recognition in continuous action streams by deep learning-based sensing fusion," IEEE Sensors Journal, vol. 18, no. 23, pp. 9660-9668, September 2018.

[17] W. Jiang, and Z. Yin, "Human activity recognition using wearable sensors by deep convolutional neural networks," Proceedings of the 23rd ACM international conference on Multimedia, pp. 1307-1310, July 2015

[18] M. M. Hassan, M. Z. Uddin, A. Mohamed, and A. Almogren, "A robust human activity recognition system using smartphone sensors and deep learning," Future Generation Computer Systems, vol. 81, pp. 307-313, April 2018.

[19] S. Harapanahalli, N. O. Mahony, G. V. Hernandez, S. Campbell, D. Riordan, and J. Walsh, "Autonomous Navigation of mobile robots in factory environment". Proceedings of Manufacturing, vol. 38, pp. 1524-1531, June 2019.

[20] A. T. Raman, V. N. Krovi, and M. J. Schmid, "Empowering Graduate Engineering Students With Proficiency in Autonomy," Proceedings of International Design Engineering Technical Conferences and Computers and Information, American Society of Mechanical Engineers Digital Collection (ASME), August 2018.

[21] F. F. Monteiro, A. L. B. Vieira, J. M. X. N. Teixeira, and V. Teichrieb, "Simulating real robots in virtual environments using NVIDIA's Isaac SDK," Proceedings in Anais Estendidos do XXI Simpósio de Realidade Virtual e Aumentada, SBC, pp. 47-48, October 2019.

[22] A. Wong, M. Famuori, M. J. Shafiee, F. Li, B. Chwyl, and J. Chung, "YOLO Nano: a Highly Compact You Only Look Once Convolutional Neural Network for Object Detection," arXiv preprint arXiv: 1910.01271, October 2019

[23] Jon Morss, Official website, "NVIDIA Jetson Nano: Collision Avoidance,"

https://www.element14.com/community/people/jomoenginer/blog/20 19/05/14/nvidia-jetson-nano-collision-avoidance, May 2019.

[24] J. Redmon and A. Farhadi, "YOLO9000: better, faster, stronger," Proceedings of the IEEE conference on computer vision and pattern recognition, pp. 7263-7271, July 2017.

[25] R. Joseph, D. Santosh, G. Ross, and F. Ali, "You Only Look Once: Unified, Real-time Object Detection," Proceedings of CVPR 2016 , April 2016.

[26] R. Girshick, J. Donahue, T. Darrell, and J. Malik, "Rich feature hierarchies for accurate object detection and semantic segmentation," Proceedings of the IEEE conference on computer vision and pattern recognition, pp. 580-587, June 2014.

[27] B. Jiang, R. Luo, J. Mao, T. Xiao, and Y. Jiang, "Acquisition of localization confidence for accurate object detection," Proceedings of the European Conference on Computer Vision (ECCV), pp. 784-799 July 2018.

[28] L. Fang, X. Zhao, and S. Zhang, "Small-objectness sensitive detection based on shifted single shot detector. Multimedia Tools and Applications, vol. 78, no. 10, pp. 13227-13245, May 2019.

[29] Wu, S., Li, X., \& Wang, X. "IoU-aware single-stage object detector for accurate localization," Image and Vision Computing, arXiv:1912.05992v4, April 2020.

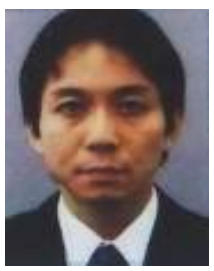

Shinji KAWAKURA Place of birth: Toyama Pref., Japan. Date of birth: July 14, 1978. Ph.D. in Environmentology, University of Tokyo, 2015, Bunkyo-ku, Tokyo, Japan. B.A. in Control System Engineering, Tokyo Institute of Technology, 2003, Meguro-ku, Tokyo, Japan. M.A. in Human-Factor Engineering, Tokyo Institute of Technology, 2005, Meguro-ku, Tokyo, Japan.

Career: Systems engineering, research for private companies. Development and verification of sensing systems for outdoor agricultural workers

Dr. KAWAKURA, Research Center for Artificial Photosynthesis (ReCAP) at Osaka City University/Osaka City, Osaka, Japan. IEEE senior member, Hong Kong Chemical, Biological\& Environmental Engineering Society (HKCBEES) senior member.

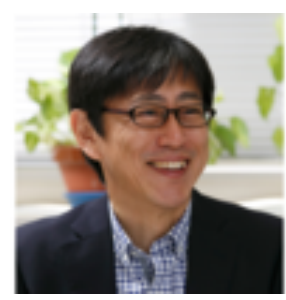

Ryosuke SHIBASAKI Place of birth: Fukuoka. Pref. Japan. Date of birth: March 1, 1958. Dr. in Engineering, the University of Tokyo, 1987, Bunkyo-ku, Tokyo, Japan. B.A in Engineering, the University of Tokyo, 1980, Bunkyo-ku, Tokyo, Japan. M.A. in Engineering, the University of Tokyo, 1982, Bunkyo-ku, Tokyo, Japan.

Career: Professor at the Center for Spatial Information Science, University of Tokyo.

Dr. SHIBASAKI, Center for Spatial Information Science (CSIS), The University of Tokyo, Meguro-ku, Tokyo, Japan, and Department of SocioCultural and Socio-Physical Environmental Studies, The University of Tokyo/Kashiwa-shi, Chiba, Japan 
